\title{
Adherence to the Mediterranean lifestyle is inversely associated with insomnia presence and severity: cross-sectional analysis in obstructive sleep apnea patients
}

\author{
Ioanna Kechribari ${ }^{1}$, Meropi Kontogianni ${ }^{1}$, Michael Georgoulis ${ }^{1}$, Kallirroi Lamprou ${ }^{2}$, \\ Antonia Kalogera ${ }^{1}$, Emmanouil Vagiakis ${ }^{2}$ and Nikos Yiannakouris ${ }^{1}$ \\ ${ }^{1}$ Department of Nutrition and Dietetics, School of Health Sciences and Education, Harokopio University, Athens, \\ Greece and \\ ${ }^{2}$ Center of Sleep Disorders, 1 st Department of Critical Care, "Evangelismos" General Hospital, Athens, Greece
}

\begin{abstract}
Insomnia is the most prevalent sleep disorder and frequently co-occurs with obstructive sleep apnea (OSA), a chronic disease characterized by repetitive pauses of breathing during sleep due to obstructions of the upper airways. The link between lifestyle and sleep quantity and quality is an area of intensive research, however data exploring associations between lifestyle habits and insomnia symptoms are still scarce. The aim of the present study was to investigate the potential association between the level of adherence to the Mediterranean lifestyle (ML), a healthy lifestyle pattern incorporating the prudent Mediterranean diet, adequate physical activity and healthy sleep habits, and insomnia presence and severity. The study sample consisted of 243 adult patients with polysomnographydiagnosed OSA. Participants' insomnia-related disorders were evaluated through the Athens Insomnia Scale (AIS), an 8-item index ranging from 0 (absence of any sleep-related problem) to 24 (severe degree of insomnia); AIS values of $>6$ were used to establish the diagnosis of insomnia. All patients were evaluated with regard to anthropometric indices and lifestyle habits, and adherence to the ML was estimated through the MEDLIFE index, a 28-item index ranging from 0 to 28, with higher values indicating greater proximity to the healthy lifestyle of the Mediterranean region. An inverse correlation was observed between the MEDLIFE index and total AIS (rho $=-0.22, p=0.001$ ), as well as most individual components of AIS, including difficulty in sleep induction (rho $=-0.14$, $\mathrm{p}=0.03$ ), awakenings during the night ( $\mathrm{rho}=-0.2, \mathrm{p}=0.008$ ), short sleep duration (rho $=-0.16, \mathrm{p}=0.01$ ), low quality of sleep $($ rho $=-0.13, p=0.05)$, low well-being during the day $(\mathrm{rho}=-0.16, \mathrm{p}=0.02)$, and low functioning capacity during the day $(\mathrm{rho}=-0.15$, $\mathrm{p}=0.02$ ). Patients with insomnia (AIS > 6), compared to those without (AIS $\leq 6)$, exhibited lower MEDLIFE index values [13 (11-15) vs. 14 (12-15), $\mathrm{P}=0.002]$, had more severe OSA as assessed by the apnea-hypopnea index (AHI) [55 (24-87) vs. 35 (17-57) events/ hour, $\mathrm{P}<0.001]$ and tended to have higher body mass index $(\mathrm{BMI})$ [35.0 (30.6-39.7) vs. $\left.32.4(29.5-38.6) \mathrm{kg} / \mathrm{m}^{2}, \mathrm{P}=0.06\right] . \mathrm{According}$ to logistic regression analysis, MEDLIFE index was inversely associated with the presence of insomnia (OR: $0.89,95 \% \mathrm{CI}$ : $0.80-0.99$, $\mathrm{P}=0.04$ ) after adjustment for age, sex, smoking, BMI, daily energy intake and AHI. In conclusion, a higher adherence to the ML is inversely associated with insomnia presence and severity in patients with obstructive sleep apnea. Future research should assess whether this association applies in other samples, as well as whether the ML could be an efficient therapeutic tool alleviating or treating insomnia symptoms.
\end{abstract}

\section{Conflict of Interest}

There is no conflict of interest. 